
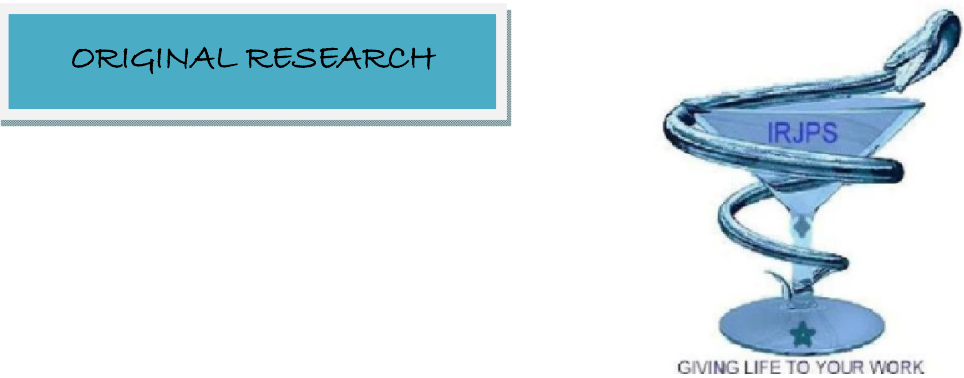

\title{
A PROSPECTIVE STUDY COMPARING ANALGESIC EFFICACY- PARACETAMOL VS COMBINATION OF PARACETAMOL\&TRAMADOL AND DRUG USE PATTERN IN POST- OPERATIVE ORTHOPAEDICS PATIENTS IN A TERTIARY CARE HOSPITAL
}

\author{
Besni.S, Chetan. J. Payyalil,Rency Ann Raju, D.R Delphin Lent*
}

Pharm.D, Department Of Pharmacy Practice, Sree Krishna CollegeOf Pharmacy and Research Centre, Trivandrum, Kerala, India

\author{
Submitted on: 14.12.16; $\quad$ Revised on: 19.12.16; $\quad$ Accepted on: 26.12.16
}

\begin{abstract}
Objective: The main objective of the study was to compare the analgesic effect of Paracetamol VsParacetamol and Tramadol by using Numeric pain rating scale, evaluate the intensity of pain in patients after orthopaedic surgeries and to analyse the drug utilization pattern in post-operative orthopaedic patients.

Methods: The comparison of analgesic effect of therapies as well as the pain intensity was assessed by using Numeric Pain Rating Scale (NRS).

Results: The data collected reveals that combination therapy (Paracetamol and Tramadol) was more effective than monotherapy(Paracetamol) and pain intensity is most predominantly observed on the first day of post operative period.

Conclusion: From the study, we got information about the drugs prescribed among post-operative orthopaedic patients, intensity of pain after orthopaedic surgeries and effectiveness of analgesics. From our analysis, we found the necessity to assess intensity of pain to select an appropriate analgesic agent to reduce the pain in post-operative orthopaedic patients.
\end{abstract}

KEYWORDS: Numeric pain rating scale, combination therapy, monotherapy, drug utilization pattern, orthopedicsurgery, pain intensity

Corresponding author: D.R Delphin Lent

Email id: besnimajeed@gmail.com
Indian Research Journal of Pharmacy and Science; 11(2016) 849-856; Journal Home Page: https://www.irjps.in 


\section{INTRODUCTION}

Pain can be defined as an unpleasant sensory and emotional experience that can result from actual or potential tissue damage ${ }^{[1]}$.Pain is the most common complaint in human beings. It can interfere with a person's quality of life and general functioning. When tissues suffer from any type of injury, cells release prostaglandins, causing hypersensitivity and results to pain. Pain can affect any system in the body such as pulmonary, cardiovascular, gastrointestinal, endocrine and immunological systems ${ }^{[1]}$. After surgical procedure, prolonged painful stimuli cause mental sufferings and physical harm to the organism and postoperative complications. Pain can cause several physiological changes and analgesia is essential for the well-being of patients. It reduces early mobilisation and disrupts sleeping. Patients undergoing orthopaedic surgeries usually have musculoskeletal dysfunctions, such as unstable fractures, deformities, joint disorders, infected or necrotic tissues, traumas or tumours. The most common surgical procedures include open and closed reduction internal fixation in fractures, arthroplasty, menisectomy, joint replacement and amputation. The goals of surgical procedures are to improve patient's body function,to hasten functional recovery,to alleviate pain and incapacity,to restore normalcy of daily functioning.Effective post-operative pain control is an essential component of the care of the surgical patient. Inadequate pain control may result in increased morbidity and mortality. Surgery suppresses the immune system and this suppression is proportionate to the invasiveness of the surgery. Good analgesia can reduce this deleterious effect.The advantages of effective postoperative pain management are patient comfort and satisfaction, earlier mobilization ,fewer pulmonary and cardiac complications, a reduced risk of deep vein thrombosis,faster recovery with less likelihood of the development of neuropathic pain, reduced cost of care $^{[4]}$.

\section{PAIN ASSESSMENT}

Acute post-operative pain management must include an appropriate assessment tool. Pain is always subjective so a person's self-reporting is the most reliable measure of pain. Systemic assessments and the use of specific scales to measure pain can contribute to adequate care and reduction in the pain pattern. There are many pain assessment scales are available to assess intensity of pain including Numeric pain rating scale (NRS), Visual analogue scale (VAS), Wong-Baker faces pain rating scale etc. NRS and VAS is more powerful tool for assessing post-operative pain intensity. They function best for the patient's subjective feeling of the intensity of pain right now. They may be used for worst, least or average pain over the last $24 \mathrm{hrs}$ or during the last week. An NRS with numbers from 0 to 10 is more practical than VAS and is easier to understand for most people. Here the patient may be asked to locate their pain on a scale of 0 to 10 , with 0 being no pain at all and 10 the worst pain they have ever felt ${ }^{[3]}$.

\section{ORTHOPAEDIC SURGERIES}

Orthopaedic surgery or Orthopaedics is the branch of surgery concerned with conditions involving the musculoskeletal system. Orthopaedics surgeons use both surgical and non-surgical means to treat musculoskeletal trauma, sports injuries, degenerative diseases, infections, tumors and congenital disorders. Some examples of orthopaedic surgeries are hand surgery, shoulder and elbow surgery, total joint reconstruction (arthoplasty), foot and ankle surgery, spine surgery, orthopaedic trauma. The most common procedures performed by orthopaedic surgeons are knee arthroscopy, shoulder arthroscopy, total hip replacement, total knee replacement, carpel turner syndrome, open reduction internal fixation, closed reduction internal fixation etc.Arthroplasty (Total joint replacement surgery) is an orthopaedic surgery where the articular surface of a musculoskeletal joint is replaced, remodelled, or realigned by osteotomy or some other procedure. An elective procedure is done to relieve pain and restore function to the joint after damage by arthiritis or some other trauma. People who have arthroplasty have substantial improvement in their joint pain, ability to perform activities and quality of life. There are different types of arthroplasty, which includes hip replacement,kneereplacement,shoulder replacement, and reverse total shoulder replacement.The procedures for arthroplasty may vary according to the type of arthroplasty. The most common type of arthroplasty is: Total knee replacement (TKR) and Total hip replacement $(\mathrm{THR})^{[6]}$.

\section{POST- OPERATIVE PAIN MANAGEMENT}

A treatment plan must always include evaluation of the following factors: age,co-morbidities (such as renal and liver disease), route of administration (the oral route may not be suitable), concurrent medications (for duplication or drug-drug interactions), laboratory abnormalities, and financial resources. Acute pain is usually managed very effectively with non-steroidalanti-inflammatory drugs (NSAIDs), acetaminophen or opioids. The goal of post-operative pain management is to relieve pain while keeping side effects to a minimum and use a variety of approaches (multimodal analgesia) to improve analgesia and decrease doses of individual agents. Paracetamol and non-steroidal anti- 
inflammatory drugs (NSAIDs) are valuable components of multimodal analgesia. Use individualized doses of analgesic(s) administered at appropriate dose intervals and titrate to patient response. A number of pain relievers are accessible and promptly regulated to control pain in postoperative patients. Different types of analgesia can be combined for additive or synergistic pain relief. The most commonly used analgesics are Paracetamol, NSAIDs and Opioids. Paracetamol and NSAIDs are used for the treatment of mild to moderate pain and opioids used for moderate to severe post-operative pain.Paracetamol, an analgesic and antipyretic, is often selected as initial therapy for mild to moderate post-operative pain and is considered first-line in several pain situations. Mechanistically, it is believed to inhibit prostaglandin synthesis in the CNS and block pain impulses in the periphery and is welltolerated at usual doses. The maximum recommended dose for patients with normal renal and hepatic function is $4000 \mathrm{mg}$ per day. Hepatotoxicity has been reported with excessive use and overdose. Regular chronic use of paracetamolhas been associated with chronic renal failure. For these reasons, the maximum dose should be reduced by $50 \%$ to $75 \%$ in patients with renal dysfunction or hepatic disease and in those who engage in excessive alcohol use.Nonsteroidal anti-inflammatory drugs (NSAIDs) have analgesic, antipyretic, and anti-inflammatory actions. These agents inhibit cyclooxygenase (COX-1 and COX-2) enzymes, thereby preventing prostaglandin synthesis, which results in reduced nociceptor sensitization and an increased pain threshold. NSAIDs are the preferred agents for mild to moderate pain in situations that are mediated by prostaglandins. They do not carry the same side effects of the opiates; therefore, although they are less potent than the narcotics, they can act as opiate-sparing agents. The development of more potent and parenteral nonsteroidalanti-inflammatory analgesics such as ketorolac has led to anincrease in their use. These drugs are particularly useful in managing the pain associated with minimally invasive surgery. However, associated side effects include peptic ulcer disease, gastrointestinal hemorrhage, renal dysfunction, altered liver function, and platelet dysfunction. These side effects limit the use of these agents in many patients during the perioperative period.Opioids are among the world's oldest known drugs. Opioids are substances that act on opioid receptors to produce morphine-like effects. Opioid drugs are predominantly central nervous system agents most often used to medically to relieve pain. Opioids are indicated for the relief of mild to severe pain, but are usually reserved for moderate to severe pain. They are useful for treatment of acute pain (such as pain following surgery). For immediate or short-term relief of moderate to severe pain, opioids are frequently the treatment of choice due to their rapid onset, efficacy and reduced risk of dependence. Carefully titrating the dose of opioids can provide for effective pain relief while minimizing adverse effects. Opioid analgesics do not cause any specific organ toxicity. The adverse effects of opioids are nausea, vomiting, drowsiness, dry mouth etc. Opioids bind to specific opioid receptors in the nervous system (neuronal cell membrane) and other tissues and produce pharmacological actions. There are three principal classes of opioid receptors, (mu, kappa and delta). The presynaptic action of opioids to inhibit neurotransmitter release is considered to be their major effect in the nervous system. These drugs have effects in both central and peripheral nervous system. Within the central nervous system, opioids have effect in many areas including the spinal cord, In the peripheral nervous system, actions of opoids in both the myenteric plexus and submucous plexus in the wall of the gut responsible for the powerful constipating effects of opioids. In peripheral tissues such as joints, opioids act to reduce inflammation. Opioid receptors are present in many regions of the nervous system that are involved in pain transmission and control, including primary afferent neurons, spinal cord, midbrain and thalamus. These drugs produce analgesia by actions on several levels of the nervous system, particularly, inhibition of neurotransmitter release from the primary afferent terminals in the spinal cord and activation of descending inhibitory controls in the midbrain. Opioids inhibit neurotransmitter release by inhibiting calcium entry, enhancing outward movement of potassium ions or by inhibiting adenocyclase enzyme which coverts adenosine triphosphate to cyclic adenosine monophoshate (cAMP). Some of the opioids are tramadol, morphine, codeine, fentanyl ${ }^{[3]}$.

MATERIALS AND METHODS

Study sight: Department of Orthopaedics, of 350 bedded cosmopolitan multispecialty hospitalsituated in Trivandrum.

Study period: Six months (December 2015-May 2016).

Study design: Prospective Observational study.

Study people: Patients from the department of Orthopaedics in cosmopolitan hospital who wereunder went orthopaedic surgeries during the study period, were included after obtaining the permission for collection of data. 
Sample Size: It was calculated for equivalence study of a continuous response variable from the two groups using the formula;

$$
N=\frac{2\left(Z_{\alpha}+Z_{\beta}\right)^{2} \bar{p}(1-\bar{p})}{\Delta^{2}}
$$

Where

$Z \alpha=1.96$ for $\infty=0.05$

$Z \beta=0.84$ for $\beta=0.80$

$$
\begin{aligned}
& \overline{\mathrm{P}}=\frac{P C+P T}{2} \\
& P C=\text { Proportion in group1 } \\
& P T=\text { Proportion in group2 } \\
& \Delta=P T-P C
\end{aligned}
$$

Minimum sample in each group is $\mathbf{6 7}$

Total 140 patients were included in the study.

\section{Inclusion criteria}

Patients above 18 years of age, Patients who underwent hip and knee arthroplasty,Patients who are conscious, able to speak and coherent, Patients who are willing to participate in the study.

\section{Exclusion criteria}

Patients who have known hepatic or renal impairment, Patients who develop allergic reactions to analgesics, Patients who are prescribed with analgesics other than Paracetamol and Tramadol.

\section{Data collection}

A written informed consent was taken in prescribed format from patient.All information relevant to study was collected from case records and directly interview the patients with the help of physician including demographic characters, anaestheticsurgical procedure, intensity of pain and prescribed analgesic plan.Postoperative pain assessment was performed with oral interviews by asking about their presence of pain at the moment of such assessment by using numeric pain rating scale.Scale varies from 0 to 10 , where 0 means absence of pain and 10 the most severe pain ever felt. Pain intensity was then categorised: no pain (0), mild pain (1-4), moderate pain (5-7), and severe pain (8-10). The assessment of pain was performed once a day, during each of following three period:Immediate post-operative period(IPO): until $24 \mathrm{hrs}$ after surgery, $1^{\text {st }}$ postoperative period $\left(1^{\text {st }} \mathrm{PO}\right)$ : from $24-48 \mathrm{hrs}$ after surgery, $2^{\text {nd }}$ post-operative period $\left(2^{\text {nd }} \mathrm{PO}\right)$ : from $48-72$ hours after surgery.The Institutional Ethics Committee of Cosmopolitan Hospital in its meeting held at Board room, reviewed and discussed the thesis protocol and was approved by the IEC committee.

\section{Data analysis}

The collected data were recorded in Microsoft excel sheet and workload is entered as numeric code. Patient prescription form is collected. For the analysis we had used SPSS (Statistical Package for Social Science) software. In inferential part we had used independent $\mathrm{t}$ - test, chi- square test appropriately. All $\mathrm{p}$ values were set at 5\%.

\section{RESULTS}

Intensity of pain in patients after orthopaedic surgeries

Percentage distribution of the sample according to post operative pain in day 1

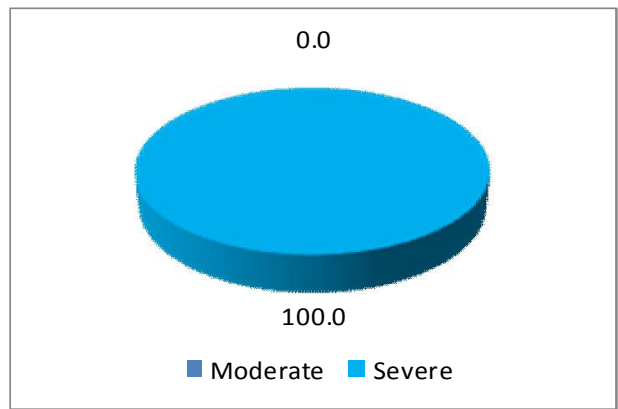

Figure 1. Graph showing

distribution of the sample according to post op pain in day 1

In terms of pain intensity, there was a predominance of severe pain in $1^{\text {st }}$ Post Operative period $(100 \%)$

Percentage distribution of the sample according to post operative pain in day 2

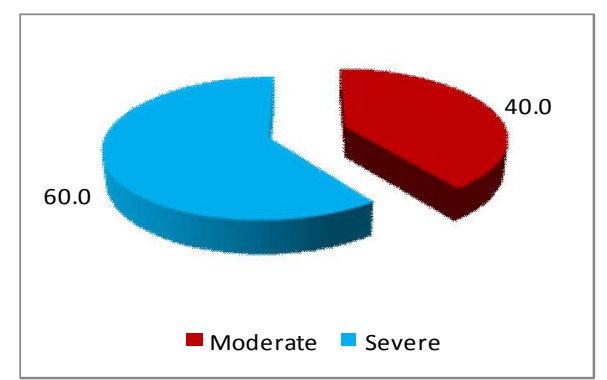

Figure 2. Graph showing distribution of the sample according to post op pain in day 2

In the $2^{\text {nd }}$ post operative period most patients had severe pain $84(60 \%)$ and $56(40 \%)$ had moderate pain. 
Percentage distribution of the sample according to post operative pain in day 3

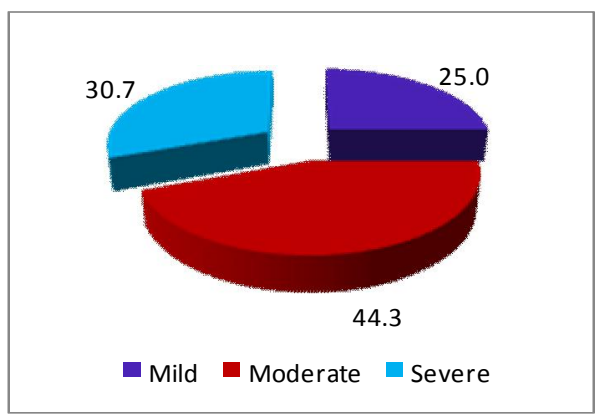

Figure 3. Graph showing distribution of the sample according to post op pain in day 3

On the $3^{\text {rd }}$ post operative period 43(30.7) patients had severe pain; 62(44.3\%), moderate pain; 43(30.7\%), mild pain.

\section{Comparison of analgesic effect of Paracetamol Vs Paracetamol\&Tramadol}

\section{Comparison of pain in day1 based on drugs}

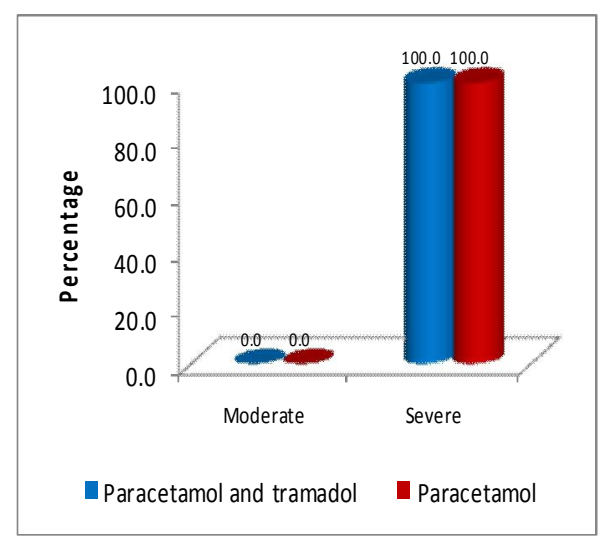

Figure 4. Graph showing distribution of comparison of pain in day1 based on drug

\begin{tabular}{|l|lr|rr|lr|r|}
\hline \multirow{2}{*}{ P a i n } & \multicolumn{2}{|l|}{ Paracetamol and tramadol } & \multicolumn{2}{l|}{ P a r a c e t a m o l } \\
\cline { 2 - 7 } & C o u n t & Percent & Count & Percent \\
\hline Moderate & 0 & 0 &. & 0 & 0 & 0 & .0 \\
\hline Severe & 6 & 9 & 100 & 0.0 & 7 & 1 & 100.0 \\
\hline Mean \pm SD & 7.9 & \pm & 0.6 & 8.6 & \pm & 0.6 \\
\hline
\end{tabular}

Table1: Comparison of pain in dayl based on drug

On day 1, 70(100\%) patients who are prescribed with Paracetamol and Tramadol had severe pain. The mean pain intensity was $7.9 \pm 0.6$. Also $70(100 \%)$ patients who are prescribed with Paracetamol alone had severe pain and the mean pain intensity was $8.6 \pm 0.6$.

\section{Comparison of pain in day 2 based on drug}

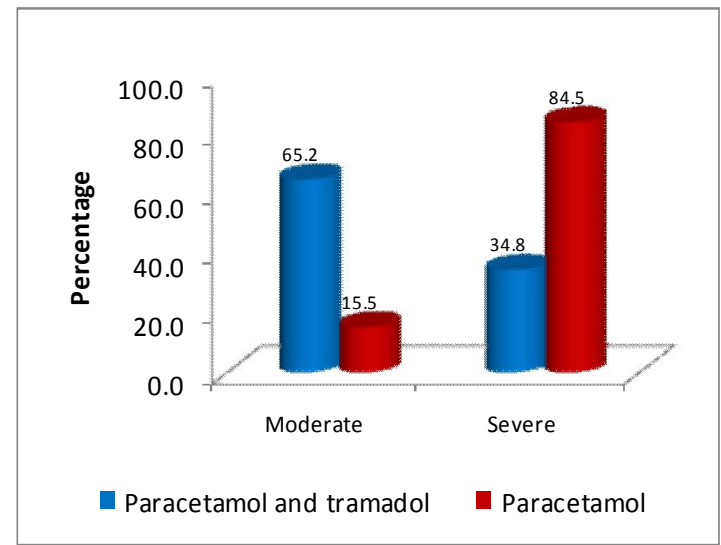

Figure 5. Graph showing distribution of comparison of pain in day 2 based on drug

\begin{tabular}{|c|c|c|c|c|}
\hline \multirow{2}{*}{$\mathrm{P}$ a i n } & \multicolumn{2}{|c|}{ Paracetamol and tramadol } & \multicolumn{2}{|c|}{ Paracetamol } \\
\hline & Count & Percent & Count & Percent \\
\hline Moderate & $4 \quad 5$ & 65.2 & 1 & 15.5 \\
\hline Severe & 2 & 34 & 6 & 84.5 \\
\hline Mean $\pm S D$ & 4 & 0 & 7 . & \pm \\
\hline
\end{tabular}

Table2: Comparison of pain in day2 based on drug

On day 2, 24(34.8\%) patients had severe pain and 45(65.2\%) patients had moderate pain in patients prescribed with Paracetamol and Tramadol whereas $60(84.5 \%)$ patients had severe pain and 11(15.5\%) patients had moderate pain in patients prescribed with Paracetamol alone.

\section{Comparison of pain in day 3 based on drug}

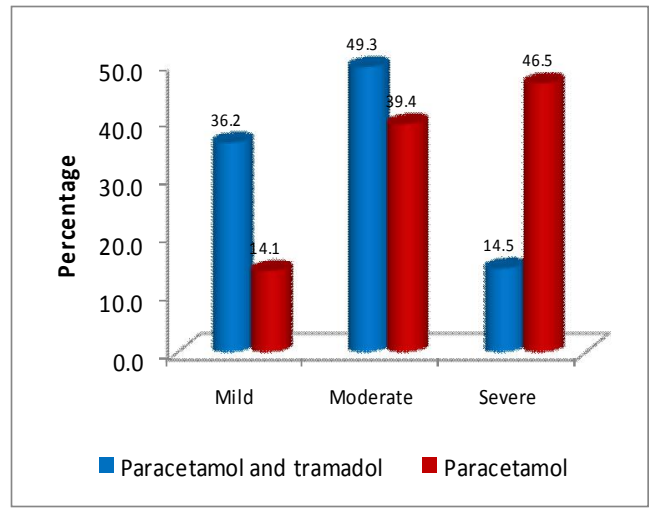

Figure 6. Graph showing distribution of comparison of pain in day 3 based on drug 


\begin{tabular}{|c|c|c|c|c|}
\hline \multirow{2}{*}{ P a in } & \multicolumn{2}{|c|}{ Paracetamol and tramadol } & \multicolumn{2}{|c|}{ Paracetamol } \\
\hline & Count & Percent & Count & Percent \\
\hline Mild & 25 & 36.2 & 10 & 14.1 \\
\hline Moderate & 4 & 49.3 & 2 & 39.4 \\
\hline Severe & 0 & 14.5 & 3 & 46.5 \\
\hline Mean \pm SD & .1 & \pm 1.4 & 5.9 & 1 \\
\hline
\end{tabular}

Table3: Comparison of pain in day3 based on drug
On day 3, 10(14.5\%) patients had severe pain, 34(49.3\%) patients had moderate pain and $25(36.2 \%)$ patients had mild pain and their mean pain intensity was $4.1 \pm 1.4$ in patients prescribed with Paracetamol and Tramadol whereas 33(46.5\%) patients had severe pain, 28(39.4\%) patients had moderate pain and $10(14.1 \%)$ patients had mild pain in patients prescribed with Paracetamol alone.

\section{Drug utilization pattern in post-operative orthopaedic patients}

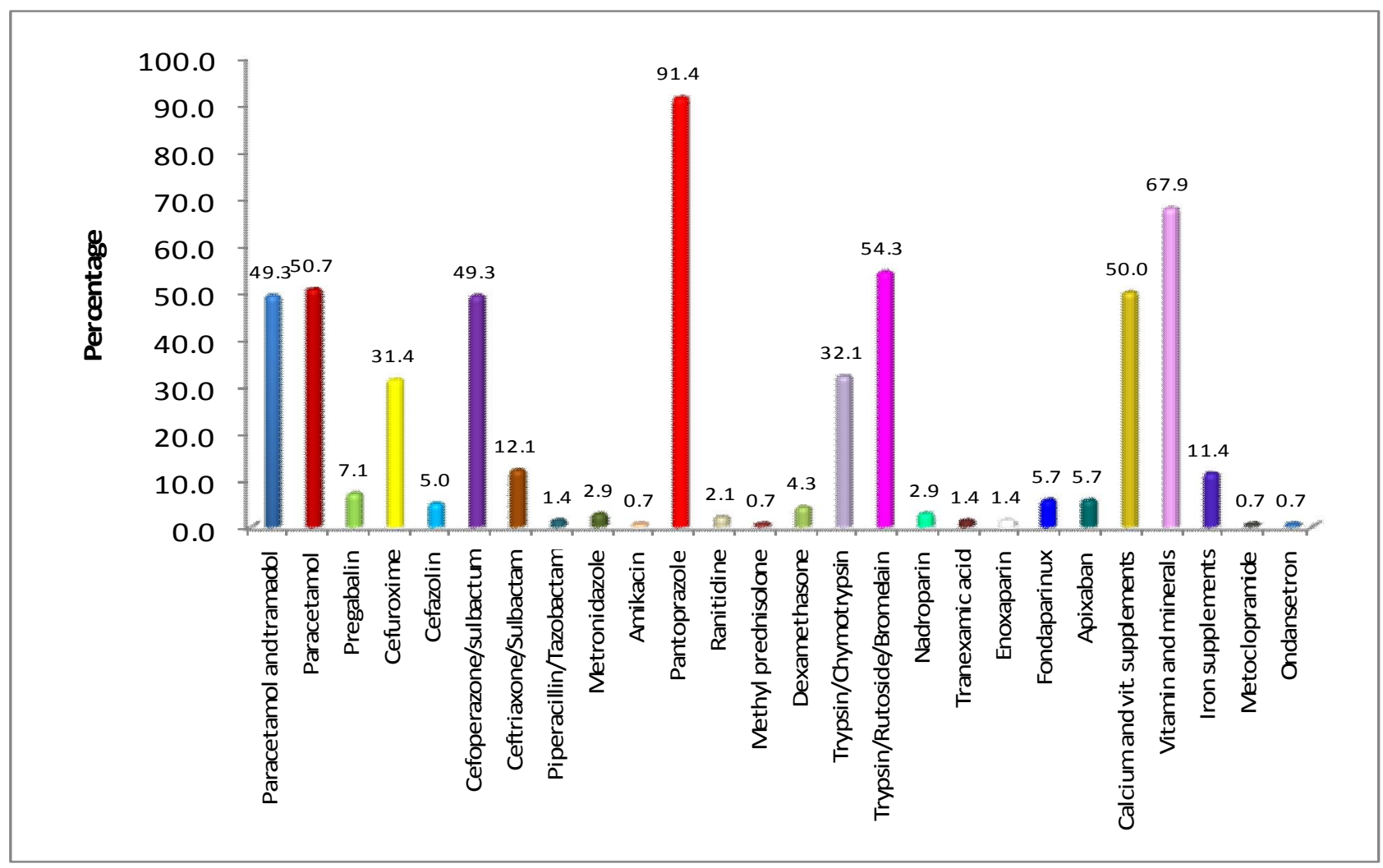

Figure 7. Graph showing percentage distribution of the sample according to drugs

Total 904 drugs were prescribed for 140 patients. Different types of drugs wereprescribed ie, 26 types of drugs. Utilization pattern of commonly prescribed drugs are shown in graph. Out of 904 medications, Pantoprazole $128(91.4 \%)$ were the highly prescribed followed by Vitamin and minerals 95(67.9\%), and Paracetamol alone and Paracetamol and Tramadol70(50.7\%). Most commonly prescribed antibiotic was Cefoperazone/Sulbactam in 69(49.3\%) patients followed by Cefuroxime44(31.4\%) and Ceftriaxone/Sulbactam17(12.1\%). The least prescribed antibiotic was Amikacin in $1(0.7 \%)$ and the commonly prescribed antiulcer drug was Pantoprazole in $98 \%$ patients. Only 2\% patients were prescribed with Ranitidine.Fondaparinux is the most commonly prescribed anticoagulant in $34 \%$ of patients followed by Apixaban (33\%) and Nadroparin(17\%).

\section{DISCUSSION}

In terms of pain intensity, there was a predominance of severe pain in $1^{\text {st }}$ post operative period $(100 \%)$ [fig.1] , in the $2^{\text {nd }}$ post operative period most patients had severe pain $84(60 \%)$ and $56(40 \%)$ had moderate pain [fig.2] and on the $3^{\text {rd }}$ post operative period 43(30.7) patients had severe pain; 62(44.3\%), moderate pain; 43(30.7\%), mild pain [fig.3]. This result is not in accordance with Maria Helena 
Barbosa et.al study. This shows that the predominance of mild pain in the $1^{\text {st }}$ post operative and $2^{\text {nd }} \mathrm{PO}(35 / 39.7 \%$ and $10 / 55.5 \%$ respectively).

In terms of analgesic efficacy,on day 1, 70(100\%) patients who were prescribed with Paracetamol and Tramadol had severe pain. The mean pain intensity was $7.9 \pm 0.6$. Also $70(100 \%)$ patients who were prescribed with Paracetamol alone had severe pain and the mean pain intensity was $8.6 \pm 0.6[$ fig. 4 \& table1].On day 2, $24(34.8 \%)$ patients had severe pain and $45(65.2 \%)$ patients had moderate pain in patients prescribed with Paracetamol and Tramadol whereas $60(84.5 \%)$ patients had severe pain and $11(15.5 \%)$ patients had moderate pain in patients prescribed with Paracetamol alone when compared to day 1 [fig.5 \& table 2].On day 3, 10(14.5\%) patients had severe pain, 34(49.3\%) patients had moderate pain and 25(36.2\%) patients had mild pain and their mean pain intensity was $4.1 \pm 1.4$ in patients prescribed with Paracetamol and Tramadol whereas 33 (46.5\%) patients had severe pain, 28(39.4\%) patients had moderate pain and $10(14.1 \%)$ patients had mild pain in patients prescribed with Paracetamol alone when compared to day 2[fig.6 \& table 3]. While comparing pain intensity on 3 consecutive post operative days there was a significant reduction in the mean pain intensity in patients who were prescribed with Paracetamol and Tramadol when compared with Paracetamol.

Utilization pattern of mostly prescribed drugs shows that out of 904 drugs, Pantoprazole128 (91.4\%) was the mostly prescribed drug followed by Vitamins and Minerals, 95(67.9\%),Paracetamol alone and Paracetamol and Tramadol70(50.7\%).[fig.7].Cefoperazone/Sulbactum $69(49.3 \%)$ is the mostly used drug in case of antibiotics whereas Pantoprazole (98\%) is the mostly used drug in anti-ulcer drugs. In case of anticoagulants, Fondaparinux (34\%) was prescribed mostly.

\section{CONCLUSION}

The present study was conducted to compare the analgesic effect of monotherapy( Paracetamol) Vs combination therapy (Paracetamol and Tramadol), to assess pain intensity after orthopaedic surgeries and drug utilization pattern in post-operative orthopaedic patients. According to our study, we concluded that combination of paracetamol and tramadol is effective than paracetamol alone and also we found that the necessity to assess intensity of pain to select an appropriate analgesic agent to reduce the pain in post-operative orthopaedic patients.

\section{REFERENCE}

1. Brian K. Alldredge, Robin L. Corelli, Michael E. Ernst, B. Joseph Gugliemlo, Pamala A. Jacobson, Wayne A. Kradjan, Bradley R. Williams. Pain and its management,Koda-Kimble \& Young's Applied Therapeutics the Clinical Use of Drugs, $10^{\text {th }}$ edition, WoltersKluwer| Lippincott Williams \& Wilkins, Philadelphia, 2005, 207-208.

2. Roger Walker, CateWhittlessa. Pain, Clinical Pharmacy and Therapeutics, $5^{\text {th }}$ edition, Churchill Livingstone Elsvier, Edinburgh London, New York,Oxford, Philadelphia, 2012, $534-541$.

3. Marie A Chisholm - Burns, Barbara G. Wells, Terry L. Schwinghammer, Patrick M. Malone, Jill M. Kolesar, John C. Rostschaffer, Joseph T. Dipiro. Pain management, Pharmacotherapy Principles and Practice, $7^{\text {th }}$ edition,New York, McGraw Hill Medical, 2008, 528 - 539 .

4. Maria Helena Barbosa, Nathalia Ferreira de Araujo, JaciaraAparecida Jesus da Silva, Thais Barbosa Correa, Tassiana Marcia Moreira, Erica Vieira Andrade.,"Pain assessment intensity and pain relief in patients post-operative orthopedic surgery".,Brazilian journal of medical sciences., Janmar- 2014;18(1):143-147.

5. MehmetZiyaYilmaz, Sarihasan BB, Kelsaka E, Tas N, Caglar Torun A, Koksal E, Kuruoglu E.,"Comparisonof the analgesic effects of paracetamol and tramadol in lumbar disc surgery"., Turkish journal of medical science., (2015); 45:438442.

6. Michael A. E. Ramsay,MD.,"Acute postoperative pain management"., BUMC proceedings., 2000;13:244-247.

7. JE. Edwards, MoQuay HJ, Moore RA., "Combination analgesic efficacy: individual patient data meta-analysis of single dose oral tramadol plus acetaminophen in acute post-operative pain"., Journal of pain and symptom management., 2002; 23:121-30.

8. Schnitzer.T., "The new analgesic combination tramadol /acetaminophen".,European journal of anaesthesiol suppl., 2003; 28:13-7.

9. Ali .M, Khan FA.,"Comparison of analgesic effect of tramadol alone and a combination of paracetamol and tramadol in day care laparoscopic surgery"., 
European journal of anaesthesiol suppl.,2009 June; 26(6):475-9.

10. Dhillon S., "Tramadol/Paracetamol fixed dose combination: A review of its use in the management of moderate to severe pain"., Clinical drug investigators., 2010; 30(10):711-38.

11. Choy Y Choy and AdnanIsquandar., "Effectiveness of tramadol/ paracetamol compared with etoricoxib as post- operative analgesia in day care surgery"., South American journal of anaesthesia and analgesia., 2014;20(3):155-159.

12. Feng-Sheng-Lin, Wen-Ying-Lin, Lai $\mathrm{CH}$, Chen CY, Lin CP, Lin TF, Sun WZ.,"Analgesic efficacy of tramadol/ acetaminophen and propoxyphene / acetaminophen for relief of post-operative wound pain”., actaanaesthesiologicataiwanica., 50(2012);4953.

13. Philip Corke.,"Postoperative pain management"., Australian prescriber., vol.36, no. 6, Dec2013.

14.Cliff K. S. Ong, Seymour RA, Lirk P, Merry AF.,"Combining Paracetamol With NSAIDs : A Quantitative Systematic Review Of Analgesic Efficacy For Acute Postoperative Pain"., International anaesthesia research society., 2010; Vol. 110,No. 4.

15.Tanveer Ahmed Khan, Sikandar Ali Zar, LubnaShakir, IftikharAhmadHotiana.,"Comparison of Analgesic Efficacy of Diclofenac Sodium, Ketorolac and Tramadol after Laparoscopic Cholecystectomy"., American journal of pharmacy and health research., 2015; Vol. 3,Issue 8. 